\title{
Peruvian Agricultural Research
}

\author{
ISSNe 2706-9397
}

Homepage: http://revistas.unjfsc.edu.pe/index.php/PeruvianAgriculturalResearch CUniversidad Nacional José Faustino Sánchez Carrión, Lima, Perú

Recibido: Diciembre 17, 2021 / Aceptado: Diciembre 30, 2021

3(2), 104-109, 2021

Comunicación corta

\section{Efecto del agua ozonizada sobre la reducción poblacional de Escherichia coli en hortalizas mínimamente procesadas}

Effect of ozonated water on the population reduction of Escherichia coli in minimally processed vegetables

\section{E. M. Caro-Degollar ${ }^{*} \mathbb{D}$, F. Fernández ${ }^{1} \mathbb{D}$, D. J. Miranda ${ }^{1} \mathbb{D}$, G. N. Vásquez $^{1} \mathbb{D}$, F. A. Bautista ${ }^{1}$, J. V. Nunja-García ${ }^{2}(\mathbb{D}$}

https://doi.org/10.51431/par.v3i2.707

\section{Resumen}

Objetivos: Determinar el efecto de la aplicación del agua ozonizada a diferentes concentraciones y tiempos de inmersión sobre la reducción poblacional de Escherichia coli (E.coli) presente en hortalizas mínimamente procesadas en un mercado peruano. Metodología: Se recolectaron e identificaron muestras de hortalizas mínimamente procesadas en ocho puestos de venta en el mercado Modelo de la Provincia de Huaral - Perú, posteriormente se determinó el recuento de E. coli en todas las muestras por triplicado y se seleccionó la muestra que presentó la media más alta; esta muestra se subdividió y se sometió a desinfección empleando agua ozonizada en los que se combinaron dos factores con tres niveles cada uno; concentración de ozono $(0,1 ; 0,5$ y 1 ppm) y tiempo de inmersión ( $30 ; 150$ y 300 segundos), finalmente se volvió a realizar el recuento de E. coli por triplicado para los nueve tratamientos a fin de ver su efecto en la población control inicial. Resultados: De las ocho muestras analizadas la más contaminada alcanzó recuentos de $4,06 \pm 0,02$ Log ufc g ${ }^{-1}$ para $E$. coli y tras su aplicación con agua ozonizada se lograron reducciones poblacionales desde $0,5 \pm 0,01$ hasta 1,76 $\pm 0,09$ Log ufc $\mathrm{g}^{-1}$ con respecto al control. Conclusiones: El tratamiento con niveles de concentración de $1 \mathrm{ppm}$ de ozono en el agua y tiempo de inmersión de 300 segundos tuvo el mayor efecto bactericida sobre la población microbiana de E. coli presente en hortalizas mínimamente procesadas en el mercado modelo de Huaral.

Palabras clave: Calidad microbiológica, coliformes fecales, higiene, vegetales

\section{Abstract}

Objectives: To determine the effect of the application of ozonated water at different concentrations and immersion times on the microbial reduction of Escherichia coli (E.coli) present in minimally processed vegetables in a Peruvian market. Methodology: samples of minimally processed vegetables were collected and identified in eight stalls in the Modelo market of the Province of Huaral - Peru, later the E. coli count was determined in all the samples in triplicate and the sample that presented the highest average; This sample was subdivided and subjected to disinfection using ozonated water in which two factors were combined with three levels in each one; ozone concentration $(0.1 ; 0.5$ and $1 \mathrm{ppm})$ and immersion time (30,150 and 300 seconds), finally the $E$. coli count was re-counted in triplicate for the nine treatments in order to see their effect in the initial control population. Results: Of the eight samples analyzed, the most contaminated reached counts of $4.06 \pm 0.02 \mathrm{Log}$ $\mathrm{cfu}^{-1}$ for $E$. coli and after its application with ozonated water, population reductions were achieved from 0.5 \pm 0.01 to $1.76 \pm 0.09 \mathrm{Log}^{\mathrm{cfu} \mathrm{g}}{ }^{-1}$ with regarding control. Conclusions: Treatment with concentration levels of $1 \mathrm{ppm}$ of ozone in water and an immersion time of 300 seconds had the greatest bactericidal effect on the microbial population of E. coli present in minimally processed vegetables in the Huaral model market.

Keywords: Microbiological quality, fecal coliforms, hygiene, vegetables

${ }^{1}$ Escuela Profesional de Ingeniería en Industrias Alimentarias, Universidad Nacional José Faustino Sánchez Carrión, Lima, Perú. ${ }^{2}$ Escuela Profesional de Ingeniería Química, Universidad Nacional José Faustino Sánchez Carrión, Lima, Perú.

*Autor para correspondencia: ecaro@unjfsc.edu.pe 


\section{Introducción}

Las hortalizas mínimamente procesadas (HMP), vienen marcando las nuevas tendencias de consumo de alimentos saludables y nutritivos; debido a sus características organolépticas, las facilidades que se presenta para su consumo y el gran aporte nutricional que brinda en fibra y vitaminas (Milte \& McNaughton, 2016; CastroIbáñez et al., 2017; Silberbauer \& Schmid, 2017; Almeida et al., 2021); sumado a esto los desórdenes nutricionales causantes de obesidad por el alto consumo de alimentos ultra procesados, excesivamente ricos en carbohidratos y grasas (Monteiro et al., 2018), promovieron la búsqueda de nuevas alternativas alimentarias saludables por parte de la población (Vidal et al., 2013), lo que trajo como consecuencia una creciente oferta de las HMP bajo una amplia variedad de presentaciones, siendo las ensaladas a base principalmente de lechuga y una porción de la mezcla de tomates, pepinillos, zanahoria y/o rabanitos (picados, rayados o en rodajas), las más populares en los mercados y mercadillos de las ciudades del Perú; lugares de venta que en su gran mayoría están formados por asociaciones de pequeños comerciantes con escasos conocimientos de manipulación de alimentos y deficientes condiciones higiénico sanitarias, el cual es agravado por la falta de acceso al agua potable, perjudicando notablemente la calidad microbiológica de los alimentos que ahí se expenden (Gil et al., 2014; Ramirez-Hernandez et al., 2020).

La presencia de enterobacterias como la $E$. coli en las HMP (Dos Santos et al., 2010, Dos Santos et al., 2021), es un indicador que alerta de un problema critico en estos productos y que demanda de acciones eficaces urgentes para su prevención o mitigación (Vilas et al., 2020), pues esta bacteria es considerada como uno de los principales microorganismos indicadores de higiene por los criterios microbiológicos peruanos (Resolución Ministerial $N^{\circ}$ 5912008/MINSA, 2008), sin embargo debemos precisar que se han identificado hasta siete patotipos perjudiciales para el hombre (FarfánGarcía et al., 2016), lo que constituye una gran problemática para la salud de la población. Por ello se han intensificado campañas para el uso de desinfectantes a base de cloro como Peruvian Agricultural Research 3(2), 104-109, 2021 una alternativa eficaz contra la presencia de patógenos en alimentos en los países en vías de desarrollo (Villeminot, 2018), no obstante uno de los inconvenientes que enfrentan los procesos que emplean al cloro, es la aparición de residuos de los sub-productos de la desinfección (SPD) en el agua y alimentos, como es el caso de los trihalometanos (Coroneo et al., 2017; Garrido et al., 2020; Marín et al., 2020) y su relación con efectos mutagénicos y cancerígenos en seres humanos (Villanueva, 2019), que alertan a la población mundial sobre los riesgos del uso del cloro.

Muchos países, conscientes del grave problema que atenta contra la salud de su población, tienen altas exigencias frente trazas de SPD (United States Environmental Protection Agency, 2020). Por ello, es importante buscar alternativas que aseguren la desinfección de las frutas y hortalizas, sin la generación de sustancias nocivas (Ali et al., 2018; Vilas et al., 2020), sobre todo en la reducción a niveles aceptables de la contaminación por E. coli en alimentos que omiten la desinfección o tratamiento térmico alguno, como es el caso de las ensaladas expendidas en los mercados del Perú.

Frente a esta problemática una alternativa es la desinfección de las HMP con ozono $\left(\mathrm{O}_{3}\right)$, una molécula triatómica de oxígeno con alta reactividad (Guzel-Seydim et al., 2004) y capacidad de dañar componentes celulares (Aslam et al., 2020; Santos et al., 2021), con el cual se ha conseguido reducir de forma significativa la presencia de microorganismos en tomates (Aguayo et al., 2014), lechugas (Akbas \& Ölmez, 2007), cebollas (Aslam et al., 2021a; Aslam et al., 2021b) y otros alimentos donde se aplicó de forma gaseosa y disuelto en agua (Vijay et al., 2021). Sin embargo, los experimentos imitan condiciones similares a las que se podrían encontrar en un lugar de expendio de las HMP ya que se inoculan cepas seleccionadas en cantidades preestablecidas parecidas a los que se encontrarían en diversos escenarios, dejando un vacío en la efectividad de la aplicación del ozono acuoso bajo condiciones de operación y contaminación real de los puntos de elaboración y expendio de las HMP como los mercados y mercadillos del Perú. Es por ello que la presente investigación tuvo como objetivo determinar 
el efecto de la aplicación del agua ozonizada a diferentes concentraciones y tiempos de contacto sobre la reducción microbiana de $E$. coli presente en las HMP en el mercado Modelo de Huaral Perú.

\section{Metodología}

El presente trabajo de investigación se realizó en los laboratorios de la Facultad de Ingeniera Agraria, Industrias Alimentarias y Ambiental de la Universidad Nacional José Faustino Sánchez Carrión (UNJFSC).

\section{Material vegetal}

Las muestras se obtuvieron de ocho puestos del mercado Modelo de Huaral - Perú. Por cada puesto se recogió bolsas de $515 \pm 25 \mathrm{~g}$, los cuales estuvieron conformados mayoritariamente por lechugas picadas y una pequeña cantidad de una mezcla de hortalizas (zanahoria rayada, tomate en rodajas y rabanito picado). Las muestras fueron identificadas, pesadas y almacenadas en refrigeración a $5 \pm 1{ }^{\circ} \mathrm{C}$ y $95 \pm 1 \% \mathrm{HR}$.

\section{Producción de agua ozonizada}

Para la producción de $\mathrm{O}_{3}$ se empleó un generador de descarga de corona (Marca OZOTECH, modelo OZ2PCS, USA) con un flujo de $1100 \mathrm{mg} \mathrm{h}^{-1}$, para ello se unió a un compresor que impulso un flujo de aire desde el ozonizador hasta un difusor de burbuja fina que se sumergió en un matraz de vidrio cerrado que contenía 1 $\mathrm{L}$ de agua tratada (libre de cloro) a $21 \pm 1^{\circ} \mathrm{C}$, el matraz presentaba un orificio pequeño cubierto con gasa para favorecer la salida de gases no solubilizados, el burbujeo con aire ozonizado se realizó hasta alcanzar la concentración de ozono en agua de cada tratamiento. Para la medición de la concentración de ozono en agua se empleó el método colorimétrico N, N-dietil-pfenilenediamina (DPD) CHECKIT ${ }^{\circledR}$ (Lovibond, M2532, Alemania).

\section{Desinfección con agua ozonizada}

A partir de la muestra de HMP del mercado con el recuento de E. coli más alto (muestra critica) se pesaron sub-muestras de $10 \mathrm{~g}$, a las que se llevó al proceso de desinfección con agua ozonizada. Cuando el agua en el contenedor de vidrio conseguía la concentración de ozono dado por cada tratamiento se procedió a añadir la submuestra de HMP y se dejó en inmersión por un lapso de tiempo. El ozonizado se realizó con nueve tratamientos en los que se combinó tres concentraciones de ozono $(0,1 ; 0,5$ y $1 \mathrm{ppm})$ y tres tiempos de inmersión (30, 150 y 300 segundos), también se añadieron tres controles a quienes se les sometió bajo las mismas condiciones de tiempos de inmersión en agua burbujeada, pero sin ozono.

\section{Recuento microbiano}

La enumeración de $E$. coli en las muestras recolectadas del mercado se realizó por triplicado por cada puesto de venta; siguiendo la metodología de la Association of Official Analytical Chemists (Food and Drug Administration, 2018), para ello se pesaron 20 $\mathrm{g}$ de las HMP y se homogenizaron con $180 \mathrm{ml}$ agua peptonada tamponada (Merck, Darmstadt, Alemania) durante 1 minuto en una bolsa estéril, posteriormente se prepararon diluciones en serie de hasta $10^{-5}$ y se extrajeron alícuotas de $1 \mathrm{ml}$ para la siembra en las películas secas hidratables de Petrifilm ${ }^{\circledR}$ Rapid E. coli/Coliform Count Plate (3M, Saint Paul, Minesota, USA), luego se llevaron a incubación de 18 a 24 horas a 42 $\pm 1^{\circ} \mathrm{C}$; los recuentos totales fueron reportados como la media del logaritmo base 10 de unidades formadoras de colonia por gramo $\left(\log \mathrm{ufc}^{-1}\right)$. Pasado el periodo de incubación se realizó la selección de la muestra más crítica es decir la que presentó mayor recuento E. coli y se sometió a la aplicación con agua ozonizada a diferentes condiciones de concentración y tiempo. Posteriormente se volvió a realizar el recuento de $E$. coli siguiendo la misma metodología empleada para el recuento previo al tratamiento de desinfección con agua ozonizada con la única variación en la dilución inicial, la cual fue de $10 \mathrm{~g}$ de HMP en $90 \mathrm{ml}$ de agua peptonada tamponada. Para determinar la reducción poblacional (RP) se restaron los valores de recuento poblacional inicial del control $\left(\mathrm{N}_{0}\right)$ y el recuento poblacional final $(\mathrm{N})$ obtenido después de la aplicación de los tratamientos con agua ozonizada.

Reducción poblacional $=\log _{10}\left(\mathrm{~N}_{0}\right)-\log _{10}(\mathrm{~N})$ 


\section{Diseño estadístico}

Para el análisis de la información se empleó el análisis de la varianza de un diseño completamente al azar con nueve tratamientos (cada tratamiento fue la combinación de tres concentraciones de ozono y tres tiempos de inmersión). La comparación de medias se realizó con la prueba de Tukey $(P=0,05)$. El software empleado fue el $\mathrm{R}$ versión 4.1.2.

\section{Resulltados y discusión}

\section{Recuento de E. coli en muestra critica}

Los recuentos de E. coli en las muestras de HMP provenientes del mercado y sin desinfectar con agua ozonizada reportó valores entre $2,51 \pm 0,03$ y $4,06 \pm 0,02$ Log ufc $\mathrm{g}^{-1}$, presentando diferencia significativa entre ellos $(P<0,05)$ (Tabla 1), resultados que son concordantes con los encontrados por Dos Santos et al., (2010) quienes reportaron valores de hasta 4,2 Log ufc $\mathrm{g}^{-1}$ en lechugas; y contradictorios con lo reportado por Dos Santos et al., (2021), quienes no observaron la presencia de $E$. coli en ensaladas de hojas mixtas de lechuga y repollo, lo cual puede deberse a que las muestras provenían de supermercados donde se aplican programas pre-

Tabla 1

Recuento de E. coli en hortalizas mínimamente procesadas en el mercado Modelo de Huaral

\begin{tabular}{cc}
\hline Muestra* $^{*}$ & $\begin{array}{c}\text { Recuento } \\
\text { (Log ufc } \mathrm{g}^{-1} \text { ) }\end{array}$ \\
\hline $\mathrm{M}_{1}$ & $3,39 \pm 0,01^{\mathrm{c}}$ \\
$\mathrm{M}_{2}$ & $4,06 \pm 0,02^{\mathrm{a}}$ \\
$\mathrm{M}_{3}$ & $2,89 \pm 0,02^{\mathrm{d}}$ \\
$\mathrm{M}_{4}$ & $2,51 \pm 0,03^{\mathrm{e}}$ \\
$\mathrm{M}_{5}$ & $3,49 \pm 0,01^{\mathrm{b}}$ \\
$\mathrm{M}_{6}$ & $2,93 \pm 0,05^{\mathrm{d}}$ \\
$\mathrm{M}_{7}$ & $3,39 \pm 0,01^{\mathrm{c}}$ \\
$\mathrm{M}_{8}$ & $3,56 \pm 0,03^{\mathrm{b}}$
\end{tabular}

${ }^{*} \mathrm{M}_{1-8}$ : cada muestra fue por triplicado y corresponde a cada puesto de verdura del mercado requisitos o sistemas de gestión de la calidad para garantizar la calidad microbiológica de las HMP. La muestra $\mathrm{M}_{2}$ presentó el mayor recuento en placa de $E$. coli $(P<0,05)$, con lo que resultó ser la muestra más crítica y la que se escogió para realizar los tratamientos de desinfección con agua ozonizada. Los resultados también demuestran que el $100 \%$ de las muestras de HMP presentan altos niveles de contaminación por E. coli resultando un indicador de las grandes deficiencias higiénico sanitarias que en los mercados del Perú se presentan.

\section{Reducción poblacional}

Las sub-muestras tomadas a partir de $\mathrm{M}_{2}$ y sometidas al tratamiento control es decir sumergidas a tiempos de 30, 150 y 300 segundos, pero sin la aplicación de ozono reportaron resultados de $3,68 \pm 0,04 ; 3,52 \pm 0,03$ y $3.45 \pm 0,01$ Log ufc $\mathrm{g}^{-1}$ respectivamente, siendo estos valores empleados como población inicial de control $\left(\mathrm{N}_{0}\right)$ para el cálculo de RP en cada nivel del tiempo de inmersión.

Los resultados de RP de las sub-muestras de HMP sometida a los tratamientos con agua ozonizada a diferentes concentraciones y tiempos de inmersión se presentan en la Tabla 2. Donde se encontró que los niveles de concentración de ozono en agua más altos y tiempo de inmersión más prolongados aplicados a las HMP reportan mayores reducciones poblacionales de E. coli, consiguiéndose disminuciones desde $0,50 \pm 0,01$ hasta $1,76 \pm 0,09 \log _{\text {ufc }} \mathrm{g}^{-1}$ con respecto al control, siendo concordantes con Hyun-Gyun et al. (2006) quienes reportan reducciones de hasta 1,09 Log para E. coli O157: H7 en concentraciones de $5 \mathrm{ppm} \mathrm{O}_{3}$ por 300 segundos y Akbas \& Ölmez (2007) quienes reportan reducciones de 1.3 Log para Enterobacteriaceae en lechugas frescas recién cortadas.

\section{Conclusiones}

El agua ozonizada utilizada a concentraciones de ozono y tiempos de inmersión crecientes, favoreció la reducción de la población microbiana. El tratamiento con niveles de concentración de $1 \mathrm{ppm}$ ozono en el agua y tiempo de inmersión de $300 \mathrm{~s}$ tuvo el mayor efecto bactericida sobre la población microbiana 
Tabla 2

Efecto de los tratamientos con agua ozonizada y tiempos de inmersión en la reducción poblacional de E. coli en hortalizas del mercado Modelo de Huaral

\begin{tabular}{cccc}
\hline Tratamiento & $\begin{array}{c}\text { Ozono } \\
(\mathrm{ppm})\end{array}$ & $\begin{array}{c}\text { Tiempo } \\
(\mathrm{s})\end{array}$ & $\begin{array}{c}\text { Reducción Poblacional } \\
\left(\text { Log ufc } \mathrm{g}^{-1}\right)\end{array}$ \\
\hline $\mathrm{T}_{1}$ & 0,1 & 30 & $0,50 \pm 0,01^{\mathrm{f}}$ \\
$\mathrm{T}_{2}$ & 0,1 & 150 & $0,62 \pm 0,02^{\text {ef }}$ \\
$\mathrm{T}_{3}$ & 0,1 & 300 & $0,82 \pm 0,03^{\mathrm{d}}$ \\
$\mathrm{T}_{4}$ & 0,5 & 30 & $0,59 \pm 0,02^{\text {ef }}$ \\
$\mathrm{T}_{5}$ & 0,5 & 150 & $0,65 \pm 0,03^{\mathrm{e}}$ \\
$\mathrm{T}_{6}$ & 0,5 & 300 & $0,99 \pm 0,04^{\mathrm{c}}$ \\
$\mathrm{T}_{7}$ & 1 & 30 & $1,45 \pm 0,04^{\mathrm{b}}$ \\
$\mathrm{T}_{8}$ & 1 & 150 & $1,51 \pm 0,03^{\mathrm{b}}$ \\
$\mathrm{T}_{9}$ & 1 & 300 & $1,76 \pm 0,09^{\mathrm{a}}$ \\
\hline
\end{tabular}

de $E$. coli presente en hortalizas mínimamente procesadas en el mercado modelo de Huaral.

\section{Referencias}

Aguayo, E., Escalona, V., Silveira, A. C., \& Artés, F. (2014). Quality of tomato slices disinfected with ozonated water. Food Science and Technology International, 20(3), 227-235. https://doi. org/10.1177/1082013213482846

Akbas, M. Y., \& Ölmez, H. (2007). Effectiveness of organic acid, ozonated water and chlorine dippings on microbial reduction and storage quality of fresh-cut iceberg lettuce. Journal of the Science of Food and Agriculture, 87(14), 2609-2616. https://doi.org/10.1002/jsfa.3016

Ali, A., Yeoh, W. K., Forney, C., \& Siddiqui, M. W. (2018). Advances in postharvest technologies to extend the storage life of minimally processed fruits and vegetables. Critical Reviews in Food Science and Nutrition, 58(15), 2632-2649. https://doi.org/10.1080/10408398.2017.1339180

Almeida, L. F. F., Novaes, T. G., Pessoa, M. C., Do Carmo, A. S., Mendes, L. L., \& Ribeiro, A. Q. (2021). Fruit and vegetable consumption among older adults: Influence of urban food environment in a medium-sized Brazilian city. Public Health Nutrition, 24(15), 4878-4887. https://doi.org/10.1017/S136898002000467X

Aslam, R., Alam, M. S., \& Pandiselvam, R. (2021) a. Aqueous Ozone Sanitization System for Fresh Produce: Design, Development, and Optimization of Process Parameters for Minimally Processed Onion. Ozone: Science and Engineering. https://doi.org/10.1080/0191 9512.2021.1984206

Aslam, R., Alam, M. S., \& Saeed, P. A. (2020). Sanitization Potential of Ozone and Its Role in
Postharvest Quality Management of Fruits and Vegetables. Food Engineering Reviews, 12(1), 48-67. https://doi.org/10.1007/s12393-01909204-0

Aslam, R., Alam, M. S., Singh, S., \& Kumar, S. (2021) b. Aqueous ozone sanitization of whole peeled onion: Process optimization and evaluation of keeping quality during refrigerated storage. LWT-Food Science and Technology, 151. https://doi.org/10.1016/j.lwt.2021.112183

Castro-Ibáñez, I., Gil, M. I., \& Allende, A. (2017). Ready-to-eat vegetables: Current problems and potential solutions to reduce microbial risk in the production chain. LWT - Food Science and Technology, 85, 284-292. https://doi. org/10.1016/j.lwt.2016.11.073

Coroneo, V., Carraro, V., Marras, B., Marrucci, A., Succa, S., Meloni, B., Pinna, A., Angioni, A., Sanna, A., \& Schintu, M. (2017). Presence of Trihalomethanes in ready-to-eat vegetables disinfected with chlorine. Food Additives \& Contaminants: Part A, 34(12), 2111-2117. https://doi.org/10.1080/19440049.2017.1382723

Dos Santos, da Silva, L. V., Lepaus, B. M., \& de São José, J. F. B. (2021). Microbial quality and labeling of minimally processed fruits and vegetables. Bioscience Journal, 37. https://doi. org/10.14393/BJ-v37n0a2021-53734

Dos Santos, Silva, N. da, Junqueira, V. C. A., \& Pereira, J. L. (2010). Microrganismos indicadores em frutas e hortaliças minimamente processadas. Brazilian Journal of Food Technology, 13(02), 141-146. https://doi. org/10.4260/BJFT2010130200019

Farfán-García, A. E., Ariza-Rojas, S. C., VargasCárdenas, F. A., \& Vargas-Remolina, L. V. (2016). Mecanismos de virulencia de Escherichia coli enteropatógena. Revista chilena 
de infectología, 33(4), 438-450. http://dx.doi. org/10.4067/S0716-10182016000400009

Food and Drug Administration. (2018). Bacteriological Analytical Manual (8th ed.). Association of Official Analytical Chemists Gaithersburg. $\quad$ https://www.fda.gov/food/ laboratory-methods-food/bacteriologicalanalytical-manual-bam

Garrido, Y., Marín, A., Tudela, J. A., Truchado, P., Allende, A., \& Gil, M. I. (2020). Chlorate accumulation in commercial lettuce cultivated in open field and irrigated with reclaimed water. Food Control, 114. https://doi.org/10.1016/j. foodcont.2020.107283

Gil, A. I., Lanata, C. F., Hartinger, S. M., Mäusezahl, D., Padilla, B., Ochoa, T. J., Lozada, M., Pineda, I., \& Verastegui, H. (2014). Fecal contamination of food, water, hands, and kitchen utensils at the household level in rural areas of Peru. Journal of Environmental Health, 76(6), 102-106. https:// europepmc.org/article/med/24645420\#abstract

Guzel-Seydim, Z. B., Greene, A. K., \& Seydim, A. C. (2004). Use of ozone in the food industry. $L W T$ - Food Science and Technology, 37(4), 453-460. https://doi.org/10.1016/j.1wt.2003.10.014

Hyun-Gyun, Y., Mee-Young, Y., Jae-Won, Y., Kwang-Deog, M., Marshall, D. L., \& DeogHwan, O. (2006). Effect of Combined Ozone and Organic Acid Treatment for Control of Escherichia coli O157:H7 and Listeria monocytogenes on Lettuce. Journal of Food Science, 71(3), M83-M87. https://doi. org/10.1111/j.1365-2621.2006.tb15636.x

Marín, A., Tudela, J. A., Garrido, Y., Albolafio, S., Hernández, N., Andújar, S., Allende, A., \& Gil, M. I. (2020). Chlorinated wash water and $\mathrm{pH}$ regulators affect chlorine gas emission and disinfection by-products. Innovative Food Science and Emerging Technologies, 66. https:// doi.org/10.1016/j.ifset.2020.102533

Milte, C. M., \& McNaughton, S. A. (2016). Dietary patterns and successful ageing: A systematic review. European Journal of Nutrition, 55(2), 423-450. https://doi.org/10.1007/s00394-015$1123-7$

Monteiro, C. A., Cannon, G., Moubarac, J.-C., Levy, R. B., Louzada, M. L. C., \& Jaime, P. C. (2018). The un Decade of Nutrition, the NOVA food classification and the trouble with ultraprocessing. Public Health Nutrition, 21(1), 5-17. https://doi.org/10.1017/S1368980017000234

Ramirez-Hernandez, A., Galagarza, O. A., Álvarez Rodriguez, M. V., Pachari Vera, E., Valdez Ortiz, M. D. C., Deering, A. J., \& Oliver, H. F. (2020). Food safety in Peru: A review of fresh produce production and challenges in the public health system. Comprehensive Reviews in Food Science and Food Safety, 19(6), 3323-3342. https://doi.org/10.1111/1541-4337.12647

Resolución Ministerial N 591-2008/MINSA, (2008). https:/www.gob.pe/institucion/minsa/normaslegales/247682-591-2008-minsa

Santos, L. M. C. D., Silva, E. S. da, Oliveira, F. O., Rodrigues, L. de A. P., Neves, P. R. F., Meira, C. S., Moreira, G. A. F., Lobato, G. M., Nascimento, C., Gerhardt, M., Lessa, A. S., Mascarenhas, L. A. B., \& Machado, B. A. S. (2021). Ozonized Water in Microbial Control: Analysis of the Stability, In Vitro Biocidal Potential, and Cytotoxicity. Biology, 10(6), 525. https://doi.org/10.3390/biology10060525

Silberbauer, A., \& Schmid, M. (2017). Packaging Concepts for Ready-to-Eat Food: Recent Progress. Journal of Packaging Technology and Research, 1(3), 113-126. https://doi. org/10.1007/s41783-017-0019-9

United States environmental protection agency. (2020). National Primary Drinking Water Regulations. https://www.epa.gov/groundwater-and-drinking-water/national-primarydrinking-water-regulations

Vidal, L., Ares, G., \& Giménez, A. (2013). Projective techniques to uncover consumer perception: Application of three methodologies to readyto-eat salads. Food Quality and Preference, 28(1), 1-7. https://doi.org/10.1016/j. foodqual.2012.08.005

Vijay Rakesh Reddy, S., Sudhakar Rao, D. V., Sharma, R. R., Preethi, P., \& Pandiselvam, R. (2021). Role of Ozone in Post-Harvest Disinfection and Processing of Horticultural Crops: A Review. Ozone: Science and Engineering. https://doi.or g/10.1080/01919512.2021.1994367

Vilas, I. A., Seró, M. A., Medà, P. C., Cordero, C. C., \& Almenar, I. V. (2020). Biopreservation against foodborne pathogens on minimally processed fruits and vegetables. Arbor, 196(795), 1-11. https://doi.org/10.3989/arbor.2020.795n1007

Villanueva, C. M. (2019). Carcinogenicity of disinfection byproducts in humans: Epidemiological studies. Encyclopedia of Environmental Health (pp. 517-527). https:// doi.org/10.1016/B978-0-12-409548-9.11191-1

Villeminot, N. (2018). Strengthening market systems that provide water and hygiene items for cholera mitigation and emergency preparedness in Haiti. Waterlines, 37(4), 307-318. https://doi. org/10.3362/1756-3488.17-00027 